\section{Mapping Population Distribution in Urban Areas: Using Sentinel-2A in Comparison with Landsat 8}

Gl_Forum 2017, Issue 1

Page: 93 - 105

Full Paper

Corresponding Author:

arthur.lehner@ait.ac.at

DOI: 10.1553/giscience2017_01_s93

\author{
Arthur Lehnerl,2 and Klaus Steinnocher ${ }^{1}$ \\ 'Energy Department, AIT - Austrian Institute of Technology, Vienna, Austria \\ 2University of Salzburg, Department of Geoinformatics, Salzburg, Austria
}

\begin{abstract}
The paper presents population disaggregation based on satellite imagery and ancillary data. The ancillary data is added stepwise in order to highlight the differences between the respective results. The methodology is applied to Landsat 8 data, and results are compared with the ones achieved by use of Sentinel-2A imagery. Part of the city of Mendoza, Argentina, was chosen as the study area. The introduction of input parameters such as land use and building heights was considered and their practical use is discussed. The paper reveals a constant improvement of the results for both Sentinel-2A and Landsat 8 data, and the advantages of Sentinel-2A, with its higher geometric resolution, over Landsat 8. These advantages include its higher spatial resolution and the resulting higher accuracy for the estimation of population. The practical usability of the proposed methodology for planning authorities and its supportive function for statistical institutes for future censuses are demonstrated.
\end{abstract}

\title{
Keywords:
}

Urban Remote Sensing, population estimation, Sentinel-2A, open data, Landsat 8

\section{Introduction}

Urbanization and urban growth have become global phenomena in recent decades. In South America, especially Argentina, the rate of urbanization is as high as $92 \%$ (EU: $73 \%$ ). Knowledge of how many people reside within an urban area and how they are spatially distributed is crucial when it comes to urban planning and discussions about public investments. Demographic data is usually derived from censuses and represented in administrative units, such as districts, municipalities or census blocks, which often vary in size. Census blocks often include areas that do not contain residential buildings or any constructions at all. Spatial analysis at a finer level is not possible due to this or other restrictions (e.g. data protection). Introducing remote sensing can partially overcome such problems by indicating where people actually live within the administrative units, and remote sensing techniques have already become a standard approach for monitoring the physical growth of urban areas (Steinnocher et al., 2011). 
Sentinel-2A data offer new perspectives for monitoring urban growth (Lefebvre et al., 2016) and the spatial distribution of population, since spatial $(10 \mathrm{~m})$ and temporal resolution are high. In combination with OSM data, potential residential areas can be identified more easily. This approach allows increased precision without additional costs for very high resolution (image) data (Gisbert, 2016) or up-to-date cadastres. Various studies confirm the usefulness of remote sensing data for disaggregation of real census data (Chen, 2002; Liverman et al, 1998). The method of spatial disaggregation has been presented for vulnerability assessment in flood-prone areas (Wurm et al., 2009) or for generating population data independently from administrative areas (Steinnocher et al., 2011). The integrated use of remote sensing and GIS data in combination with spatial disaggregation allows for the creation of refined, functional, 3D city models. The comparison between extrapolation and spatial disaggregation methods shows the difference between the use of generalized population information and punctual population data (Taubenböck et al., 2007). Spatial disaggregation based on remote sensing may also support conventional census data in the future (Wurm et al., 2011). The present study discusses the spatial disaggregation of population based on Sentinel-2A imagery and ancillary data. Previous studies (Dong et al., 2010; Iisaka \& Hegedus, 1982; Wu \& Murray, 2007) dealing with the estimation of population by means of remote sensing and ancillary data have demonstrated the usability of Landsat data for dasymetric mapping. In this study, we test the hypothesis that information added step-wise leads to continuous improvement of results. Moreover, we demonstrate that the satellite imagery with higher spatial resolution (Sentinel-2A) actually does lead to better results when compared to satellite imagery with coarser spatial resolution (Landsat 8).

\section{Study area and data basis}

\section{Study Area}

The city of Mendoza is located in western Argentina, South America (Figure 1). The climate is arid, and precipitation is about $200 \mathrm{~mm}$ p.a. Agricultural fields and urban green areas are irrigated. The central district of the city of Mendoza was chosen as the study area. 

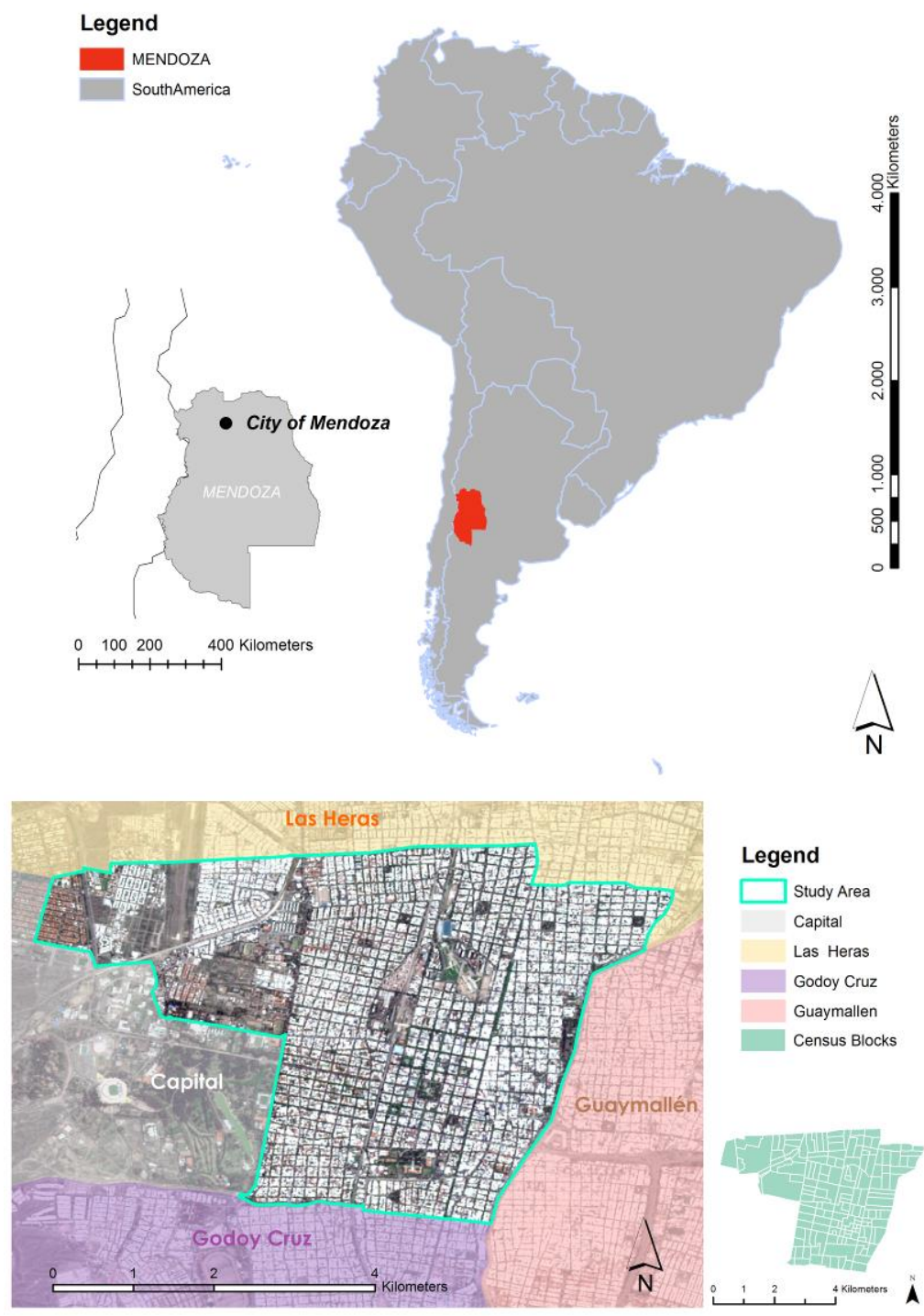

Figure 1: Location of the study area

This district is representative of other cities in South America since it contains typical urban structures like residential and industrial buildings of different sizes and heights, parks, informal settlements, apartment complexes, suburban areas, development areas and a dense city centre.

\section{Data}

For the study area of Mendoza, a geodatabase was set up consisting of Sentinel-2A (Level1C), Landsat 8 (LT1) and ancillary data. Table 1 provides an overview of all input data used. 
Because all input data was originally in digital format but not necessarily geocoded, the format of particular input data had to be modified or vectorized. Challenges arose because the land use plan, the sub-districts layer and the census blocks layer, for example, do not share the same administrative limits. Since the census blocks provided the most exact spatial data for spatial reference, the sub-districts map and the land use plan were adjusted accordingly. Both Sentinel-2A and Landsat 8 data were acquired around the same time of year and at about the same time of day. The Landsat 8 data used for this study is a corrected Level 1 Terrain - a precise ortho-corrected product (LT1). The spatial resolution of Landsat 8 is 30 metres (bands: visible spectrum: 2, 3, 4; NIR: 5; SWIR). In this study, bands 2, 3, 4 and 5 were used. The spatial resolution of Sentinel-2A is 10 metres. All data used for this study is freely available.

Table 1: Overview of Input Data

\begin{tabular}{|c|c|c|}
\hline \multicolumn{3}{|l|}{ Input Data } \\
\hline Data Type & $\begin{array}{l}\text { Data } \\
\text { Format }\end{array}$ & Time of generation \\
\hline Sentinel-2A image 19HED &.$j \mathrm{p} 2$ & 2016-01-22, 14:41 \\
\hline $\begin{array}{l}\text { Landsat } 8 \text { image } \\
\text { LC82320832016040LGN00 }\end{array}$ & .tif & 2016-02-09, 14:28 \\
\hline OpenStreetMap (OSM) & Esri.shp & $\mathrm{n} / \mathrm{a}$ \\
\hline Land use plan & .pdf & 2014 \\
\hline Sub districts map & .png & $\mathrm{n} / \mathrm{a}$ \\
\hline Census blocks &. $\mathrm{kmz}$ & $\mathrm{n} / \mathrm{a}$ \\
\hline $\begin{array}{l}\text { Building blocks (modified } \\
\text { cadastre) }\end{array}$ & dwg & $\mathrm{n} / \mathrm{a}$ \\
\hline Population data & .xlsx & 2010 \\
\hline
\end{tabular}

\section{Methodology}

The objective of the study was to investigate whether Sentinel-2A data can be used for spatially distributing the population in the built-up areas of one entire region, and whether ancillary data would be suitable to improve the result. The hypothesis that the use of satellite imagery with higher spatial resolution (Sentinel-2A) leads to better results when compared to satellite imagery with coarser spatial resolution (Landsat 8) was tested. The method chosen for this study was spatial disaggregation, which is based on the assumption that data, provided globally for an entire region, are distributed within the region by means of local parameters. The spatial distribution is performed by a weighted sum. A strong dependency between the global and the local parameters is a precondition for this approach (Liverman et 
al., 1998). The global parameter for the spatial disaggregation is the total population of the region, while the local parameter is the housing density derived from satellite imagery. The local population distribution is estimated by applying housing density as a proxy for population density. We can formalize this approach as follows:

$$
\begin{aligned}
& \text { Pdens }=\mathrm{k} * \text { Hdens } \\
& \text { Pop }=\sum \mathrm{Ai} * \mathrm{k} * \text { Hdensi }
\end{aligned}
$$

Pdens and Hdens are the population and the housing densities respectively. Pop represents the total population of the region and Ai corresponds to the area of the housing density $\mathrm{i}$. The factor $\mathrm{k}$, representing the relationship between population and housing density, can be derived by solving equation (2). The local population density is calculated from equation (1) (Liverman et al., 1998; Steinnocher et al., 2011). This method of population disaggregation has been used successfully in previous spatial disaggregation studies (Steinnocher et al., 2011, 2006).

One approach to improve the estimation of population is to introduce the third dimension in the form of building heights (Alahmadi et al., 2013; Gisbert, 2016). The information regarding building heights can be used as a weighting factor by calculating the volume of buildings. In our case, information about building heights is available only for entire land use zones. Within each land use zone, a maximum height for buildings is given. Thus the building blocks of different land use zones share the same height according to the land use plan, as three different land use zones (residential, commercial and central) feature the same maximum height.

Our study uses Sentinel-2A and Landsat 8 for estimating housing density and ancillary data. These data include the OSM layer, information about the land use, and information about the building heights derived from the land use plan. The information about the land use and height is used as weighting factors. For our study case, we applied the method using a Normalized Difference Vegetation Index-based (NDVI) settlement layer derived from Sentinel-2A data, and population data for a census block level that was aggregated on to district level. The same methodology was then applied on selected Landsat 8 data in order to compare the results achieved with Sentinel-2A data. Further explanations do not explicitly differentiate between Sentinel-2A and Landsat 8 data, since the methodology is applied similarly and is generally independent of the spatial resolution of one satellite's sensor(s).

\section{Transferability}

The actual study area is a part of the central district of the city of Mendoza, covers an area of $18.01 \mathrm{~km} 2$, and consists of 162 census blocks. The total number of people residing in the area is 102,325 . Since this study will be the initial point for a city-wide spatial disaggregation in the future, crucial elements were the collection of experience and ensuring the transferability of all computational steps. 


\section{Step-by-Step approach}

The use of different input data and the demand for transferability necessitated a distinct way to add information to the spatial disaggregation model: a step-by-step approach was chosen. Each additional input layer (the OSM and land-use layers, for example) was gradually added, and the particular result at each step (LoA) was compared to the ground truth represented by the official census blocks. The NDVI derived from the Sentinel-2A image was used to generate the settlement layer. The reclassifications were done using the quantile reclassification method. The threshold is defined by choosing the lowest quantile of the reclassification as non-built-up area and the other upper quantiles as built-up areas. Due to spectral similarity, bare soil is also included in the category "built-up". However, if the information for building blocks or building footprints is available, it is not necessary to add further steps to distinguish between bare soil and other non-built-up area, or bare soil and built-up area.

The first step was to set up a binary NDVI-based settlement layer, consisting of two values:

- $\quad 0=$ non-built-up area

- $\quad 1$ = built-up area

Within the second step, the NDVI was also used as a basis for a settlement layer that was reclassified into 5 classes (NDVISL_5):

- $\quad 0=$ non-built-up area

- $\quad 1-4=$ different densities of built-up area

Each processing step provided the basis for the next step. Therefore, the third step (LoA 3) used the NDVI-based settlement layer (NDVISL_5) and additional information about the land use in order to exclude non-residential areas or areas that do not include buildings in general (parks, development areas, airports). For the fourth step (LoA 4), this information (LoA 3) was used and merged with the OSM layer in order to exclude streets as inadequate space for settlement. For the next step, the LoA 4 was merged with the information from the cadastre. This step (LoA 5) allowed us to distribute population to building blocks only. In the final step (LoA 6), information about the land use derived from the land use plan was used as a weighting factor in order to reduce overestimation within densely built-up areas that are characterized mainly by commercial activity. In addition, the NDVI-based settlement layer was reclassified and reversely weighted. This means that the value of areas showing a high degree of impervious surface was reduced and the value of less dense areas increased (Table 2). Additionally, the resulting layer was merged again with the building blocks layer. The methodology resulted in 6 processing steps, listed in Table 3. The result of every step represents a certain level of accuracy (LoA). The sequences for the processing steps are shown in a flowchart (Figure 2). Finally, the disaggregation results are validated against detailed reference data from the census. 


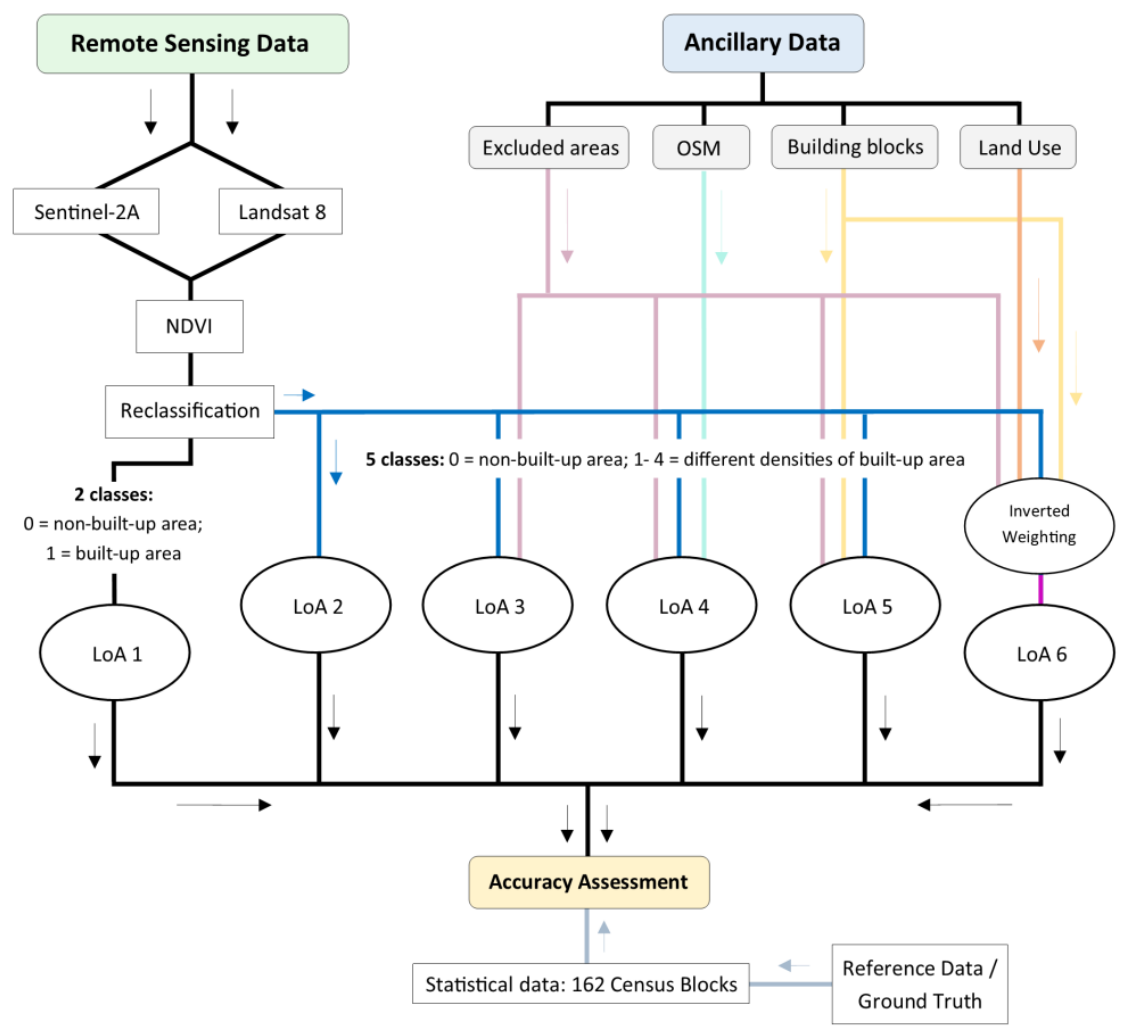

Figure 2: Flowchart for the processing steps

Table 2: Overview of Weighting for LoA 6

\begin{tabular}{|c|c|c|c|}
\hline \multicolumn{4}{|c|}{ Weighting } \\
\hline $\operatorname{LoA}$ & Input data & Weighted classes & Weights \\
\hline \multirow{3}{*}{6} & \multirow{3}{*}{ Land Use: 3 classes } & Low-density residential area & 3 \\
\hline & & $\begin{array}{c}\text { Medium-density residential } \\
\text { areas }\end{array}$ & 2 \\
\hline & & Commercial Areas & 1 \\
\hline \multirow{5}{*}{6} & \multirow{5}{*}{$\begin{array}{l}\text { NDVI-based settlement } \\
\text { layer: } 5 \text { classes }\end{array}$} & \multirow{5}{*}{$\begin{array}{l}\text { Building density }+ \\
\text { Excluded areas }\end{array}$} & 4 (low) \\
\hline & & & 3 (medium low) \\
\hline & & & 2 (medium high) \\
\hline & & & 1 (high) \\
\hline & & & 0 (excluded areas) \\
\hline
\end{tabular}




\section{Quality Assessment}

For the quality assessment, the Total Relative Error (TRE) (3) was calculated using the relative deviation between the results of the spatial disaggregation and the ground truth represented by the official census blocks.

TRE $=\sum(\mid$ Popref - Popdis $\mid *$ Ai $) \div \sum$ (Popref*Ai)

Popref represents the population count per census unit derived from statistical data of the regional statistical institute (DEIE - La Dirección de Estadísticas e Investigaciones Económicas de la Provincia de Mendoza). PopDis represents the estimated population that was calculated by applying the spatial disaggregation methodology presented here. The area Ai of each census block was attached as a weight to the absolute difference between the reference population data Popref and the estimated population Popdis of each census block. The calculation of the absolute difference is done on a census-unit basis, and is carried out for each census unit of the study area. The Total Relative Error is given as a percentage and presented for all LoAs in Table 3.

Table 3: Overview of the results

\begin{tabular}{|c|l|c|c|}
\hline \multicolumn{2}{|c|}{ Results } \\
\hline LoA & Description of Input layers & $\begin{array}{c}\text { Total Relative Error: } \\
\text { Sentinel-2A }\end{array}$ & $\begin{array}{c}\text { Total Relative Error: } \\
\text { Landsat 8 }\end{array}$ \\
\hline 1 & $\begin{array}{l}\text { NDVI-based settlement layer: } \\
\text { binary (0-1) }\end{array}$ & $80.2 \%$ & $82.7 \%$ \\
\hline 2 & $\begin{array}{l}\text { NDVI-based settlement layer: 5 } \\
\text { classes (0-4) (NDVISL_5) }\end{array}$ & $84.1 \%$ & $56.8 \%$ \\
\hline 3 & $\begin{array}{l}\text { NDVISL_5, excluded areas (parks, } \\
\text { development areas) }\end{array}$ & $48.6 \%$ & $58.3 \%$ \\
\hline 4 & $\begin{array}{l}\text { NDVISL_5, excluded areas, OSM } \\
\text { layer }\end{array}$ & $\begin{array}{l}\text { NDVISL_5, excluded areas, } \\
\text { building blocks }\end{array}$ & $37.5 \%$ \\
\hline 6 & $\begin{array}{l}\text { NDVISL_5, excluded areas, } \\
\text { building blocks, land use }\end{array}$ & $31.3 \%$ & $34.9 \%$ \\
\hline
\end{tabular}

It is worth underlining that the results table is not intended to provide an absolute or comprehensive metric of map accuracy. It represents an indicator of quality for the methodology used. 


\section{Results}

The spatial disaggregation was performed for all 6 processing steps. The results show a constant improvement for the Total Relative Error (Table 3).

\section{Sentinel-2A}

The Total Relative Error decreases when input parameters that modify the spatial distribution of the population are added. In Figure 3, the results for the 5th and 6th processing steps (LoA 5 and LoA 6) are presented. Green areas represent census blocks that were deemed to have been estimated acceptably and also include blocks that were slightly over- or underestimated $(-0.3-0.3)$. The relative difference represents the factor of the under- or overestimation. An overestimation for a census block (orange-red) that has the factor 1 means that twice as many people were estimated as actually counted according to the official census data. We recognize areas that present medium underestimation (blue) and higher overestimation (orange and red) of population, while areas in yellow show a medium overestimation of -0.4 to -0.9 .
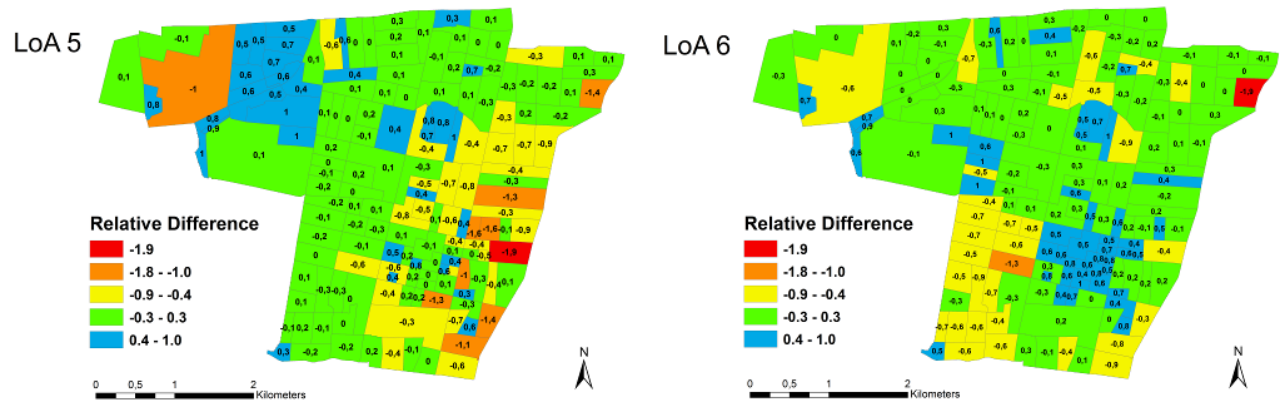

Figure 3: Results for Sentinel-2A; left: LOA 5 - NDVISL_5, excluded areas, building blocks; right: LoA 6 NDVISL_5, excluded areas, building blocks, and land use 

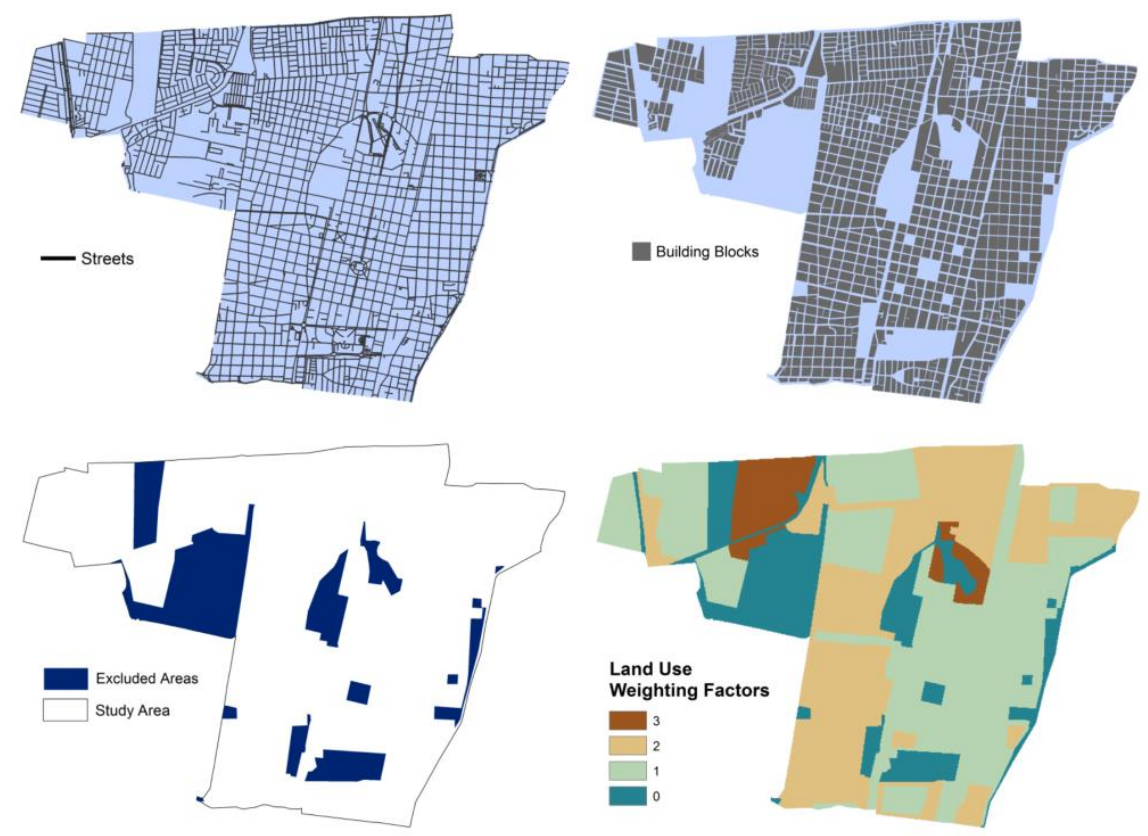

Figure 4: Input data: OSM (top left), building blocks (top right), excluded areas (bottom left), land use (bottom right)

Figure 4 shows the input data used (excluded areas, land use, OSM, building blocks). We consider this LoA an expert layer because the information of the land use as well as the NDVI-based settlement layer was weighted in response to the previous results (LoA 3 - LoA 5). In Figure 6, the results for LoA 6 of both Sentinel-2A and Landsat 8 are compared.

\section{Landsat 8}

The Total Relative Error generally decreases when input parameters that modify the spatial distribution of the population are added (Figure 5). However, LoA 2 and LoA 4 show a slight decrease, since their Total Relative Error increases compared to the corresponding previous Level of Accuracy (LoA) (Table 3).

The Total Relative Error of results achieved by means of Landsat 8 is higher (Table 2) than that for the results from Sentinel-2A. The Total Relative Error of LoA 6 Landsat 8 is 34.8\%. 


\section{LoA 5 (Landsat 8)}

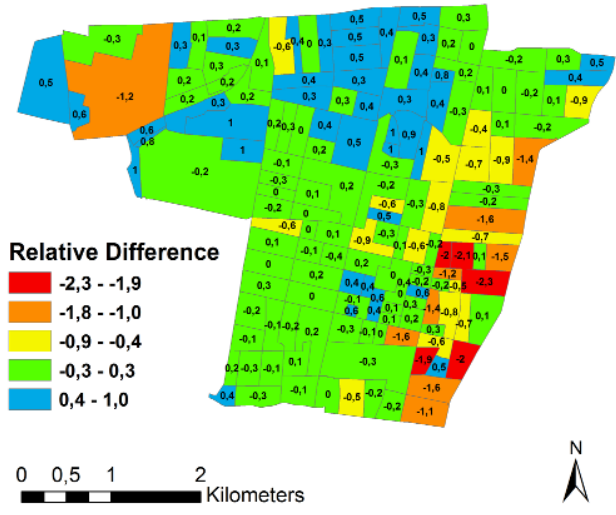

LoA 6 (Landsat 8)

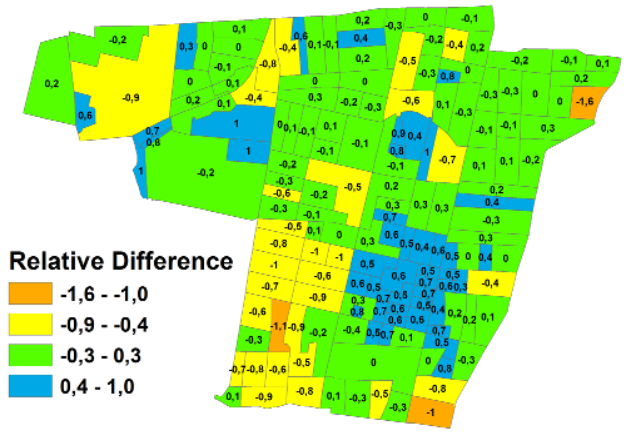

$\stackrel{N}{\Lambda}$

Figure 5: Left: LOA 5 - NDVISL_5, excluded areas, building blocks; right: LOA 6 - NDVISL_5, excluded areas, building blocks, land use

\section{LoA 6 (Sentinel-2A)}

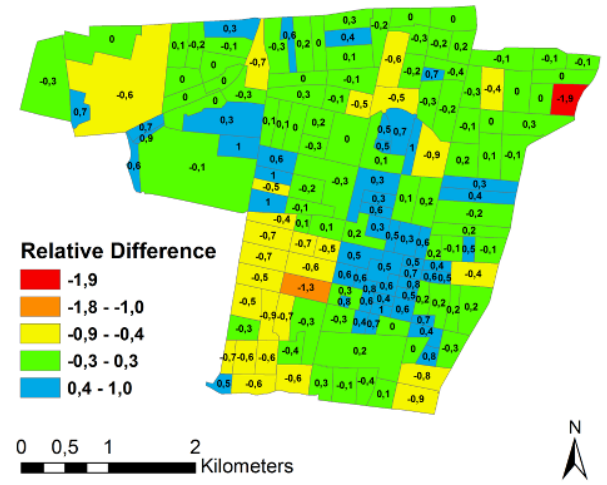

\section{LoA 6 (Landsat 8)}

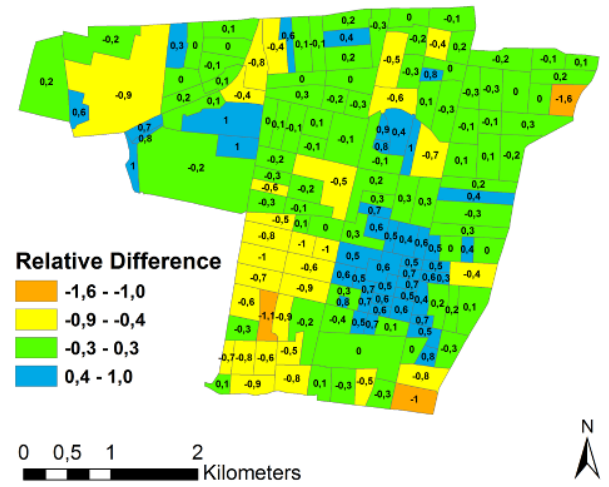

Figure 6: Comparison of Sentinel-2A and Landsat 8 results; left: LoA 6 - Sentinel-2A; right: LOA 6 Landsat 8

\section{Discussion}

In order to better understand large deviations, we compared the results with the land use plan, very high resolution satellite image data and Google Street View. Areas that show an overestimation of population are characterized by sparse building distribution or correspond to very densely built-up areas. The sparse building distribution is found mainly in the developing areas in the north-west of our study area. Within this zone, the area surrounding buildings is very dry soil, without vegetation. Thus it was partly misclassified as built-up area.

The dense built-up areas occur mainly in the south-east part of the study area, close to the old city centre. They are characterized by high commercial activity and large-scale impervious surfaces, such as shopping centres and carparks in particular. The NDVI-based settlement 
layer shows high values for impervious surfaces within these areas. Using our methodology, these characteristics lead to overestimation of population (LoA 2 to LoA 5) within the respective census blocks.

In order to address the problem of the missing information for actual building heights, we used the land use as a weighting factor in our calculations (LoA 6). This had the effect of reducing the overestimation. Underestimated areas in the north-west of the study areas also showed an improved estimation result. However, other residential areas that were calculated acceptably before now appeared to be slightly overestimated. This 6th processing step (LoA 6) could decrease Total Relative Error but partly at the expense of previously acceptable estimates for census blocks. Because data about land use, building heights or even population density were only available at larger and coarser scale or covered large areas, these input parameters could not improve the Total Relative Error significantly. The desirability of using height information as a suitable input parameter for spatial disaggregation strongly depends on the particular scale of the land use and land development plans or other maps from which the parameter is derived. The introduction of the 3rd dimension in the form of actual building heights would add information. TanDEM-X, Google StreetView or site inspections are possible sources for the acquisition of such information. However, the added information does not necessarily lead to improved results.

The computed LoA 4 for Landsat 8 shows a Total Relative Error of 58.3\% (Table 3), which means an increase of $8.5 \%$ of the Total Relative Error compared with the corresponding previous Level of Accuracy, LoA 3. This leads back to the fact that the street network, originally a vector dataset and now rasterized to 30 metres (spatial resolution of Landsat 8), reduces information on the respective settlement layer. The settings for the transformation were chosen according to the actual, visibly recognizable, layout of the street network represented in the grid of settlement layer no. 3 (LoA 4). This caused a loss of information. Pixels of built-up area were overlain by pixels from the street network (considered as nonbuilt-up area); they were therefore not considered a candidate area for population distribution.

Contrary to the case using Sentinel-2A, the lower resolution of Landsat 8 and the influence of the street network cannot lead to further improvement of the Total Relative Error. Spatial elements of the urban environment such as street corridors or building blocks are better represented within Sentinel-2A data and can be distinguished more easily from each other.

\section{Conclusion}

This paper described the use of Sentinel-2A data for population estimation. The global parameter for spatial disaggregation was the total population of the study area within the city of Mendoza, Argentina. The local parameter was the housing density derived from Sentinel2A imagery. Additional input layers, such as OSM data, land use and building heights, were considered and their practical use was critically discussed. It has been shown that gradually adding information could improve the results, while the resolution of input layers like land use or building heights derived from the land use plan proved to be limiting factors. The introduction of other sources for actual building height information was suggested. 
To conclude, the study revealed the practical usability of the proposed methodology for planning authorities and could be used to support statistical institutes for future census tasks. The comparison of Sentinel-2A with Landsat 8 revealed the importance of Sentinel-2A data and its usability for the future. The study showed that Sentinel-2A imagery could be used to complement Landsat 8 data. Sentinel-2A imagery, with its higher geometric resolution, has advantages over Landsat 8, including its higher spatial resolution and the resulting higher accuracy for the estimation of population. The study provides a model for further studies on the spatial disaggregation of population based on satellite imagery on a city-wide scale.

\section{References}

Alahmadi, M., Atkinson, P., \& Martin, D. (2013). Estimating the spatial distribution of the population of Riyadh, Saudi Arabia using remotely sensed built land cover and height data. Computers, Environment and Urban Systems, 41, 167-176.

Chen, K. (2002). An approach to linking remotely sensed data and areal census data. International Journal of Remote Sensing, 23(1), 37-48.

Dong, P., Ramesh, S., \& Nepali, A. (2010). Evaluation of small-area population estimation using LiDAR, Landsat TM and parcel data. International Journal of Remote Sensing, 31(21), 5571-5586.

Gisbert, F. J. G. (2016). Una aproximación volumétrica a la desagregación espacial de la población combinando cartografía temática y datos LIDAR. Instituto Valenciano de Investigaciones Económicas, SA (Ivie).

Iisaka, J., \& Hegedus, E. (1982). Population estimation from Landsat imagery. Remote Sensing of Environment, 12(4), 259-272.

Lefebvre, A., Sannier, C., \& Corpetti, T. (2016). Monitoring Urban Areas with Sentinel-2A Data: Application to the Update of the Copernicus High Resolution Layer Imperviousness Degree. Remote Sensing, 8(7), 606.

Liverman, D., Moran, E. F., Rindfuss, R. R., \& Stern, P. C. (1998). People and pixels: linking remote sensing and social science.

Steinnocher, K., Köstl, M., \& Weichselbaum, J. (2011). Grid-based population and land take trend indicators-new approaches introduced by the geoland 2 core Information Service for Spatial Planning. In NTTS conference, Brussels.

Steinnocher, K., Weichselbaum, J., \& Köstl, M. (2006). Linking remote sensing and demographic analysis in urbanised areas. In Proceedings: First workshop of the EARSeL SIG on urban remote sensing. Berlin, Germany.

Taubenböck, H., Roth, A., \& Dech, S. (2007). Linking structural urban characteristics derived from high resolution satellite data to population distribution. Urban and Regional Data Management, 35-45.

Wu, C., \& Murray, A. T. (2007). Population estimation using Landsat enhanced thematic mapper imagery. Geographical Analysis, 39(1), 26-43.

Wurm, M., Taubenböck, H., Goebel, J., Esch, T., \& Wagner, G. G. (2011). Zensus der Zukunft? Standort-Zeitschrift Für Angewandte Geographie, 35(4), 169-175.

Wurm, M., Taubenböck, H., Krings, S., Birkmann, J., Roth, A., \& Dech, S. (2009). Derivation of population distribution for vulnerability assessment in flood-prone German cities using multisensoral remote sensing data. In SPIE Europe Remote Sensing (p. 74781K-74781K-12). International Society for Optics and Photonics. 